\title{
ANALYSIS OF GNSS DATA USING PRECISE POINT POSITIONING TECHNIQUE FOR THE DETERMINATION OF PERMANENT STATION IN ROMANIA
}

SORIN NISTOR - lecturer Ph.D. - University of Oradea, sonistor@uoradea.ro

AURELIAN STELIAN BUDA - lecturer Ph.D. eng. - University of Oradea, budaaurelian68@yahoo.ro

\begin{abstract}
To obtain the coordinates by means of precise point positioning (PPP) technique we need to use the undifferenced GPS pseudocode and carrier phase observations but to obtain the "precise" positioning we need precise orbit and clock data too. This products and other information for obtaining the results by using PPP technique on a centimeter level accuracy can be downloaded from different locations, but the most reliable satellite ephemerides and clock correction are available from International GNSS Service (IGS).

In the PPP analysis we determined the parameters such as the receiver clock error, ionospheric delays code biases, code multipath and the total neutral atmosphere delay of the observations. For the determination of the permanent station coordinates, using the PPP technique, we used precise orbit and clock solutions to enable absolute positioning of a single receiver.

In this article we present the results obtained by using the PPP technique on the permanent station Oradea, from which we can conclude that the PPP technique can be used for different GNSS application.
\end{abstract}

Keywords: IGS, positioning, precise orbit, clock solutions

\section{Introduction}

The principles of relative positioning are the most common concept used by commercial software. Jet Propulsion Laboratory (NASA) pioneered a new technique in the late 1990's in which they managed to obtain precise positioning without using the concept of relative positioning. This technique was called precise point positioning (PPP) and the mathematical model was introduced in their software called GIPSY/OASISII [1].

One of the most important questions regarding the GPS processing is:

What are the sources of the errors?

Because the GPS point positioning is using only one receiver the limitation of this technique appears because of unmodelled errors and biases.

The concept of precise point positioning (PPP) is currently associated with global networks and to enable absolute positioning of a single receiver we need precise orbit and clock solutions [2]. The concept of "precise" in this technique appears because when we are enabling the data processing we use precise a priori information, such as satellite orbits as well as clock errors and because the resulting position coordinates are precise and accurate [3].

The PPP can be improved especially by doing extensive research on ambiguity fixing which is the goal for the next ten years for GNSS (Global Navigation Satellite System) research and applications [4].

The zero-difference (ZD) ambiguity represents the major problem for the PPP even if we talk about a satellite-receiver pair or a single-difference (SD), the nature of the ambiguity between two satellites not being an integer value. This problems arise from the uncalibrated phase delays (UPD) originating in the receiver and the satellite [5].

Therefore long integration or observation times are required for PPP. High-rate PPP is capable of obtaining the same high accuracy as GPS relative positioning techniques, but requires no fixed datum station [6]. 
The primary advantage of PPP is that a user does not need observation data from other receivers to determine the position of their own receiver [3].

A major question appears like "what datum are the coordinates in?" This question arises because in the PPP technique no reference station is required. The datum of PPP is hidden in the satellites coordinates - the satellite reference frame (datum) will be the unknown ground site reference frame. We can conclude that to obtain the coordinates in a different reference frame the user needs to perform a usually straightforward coordinate transformation [1].

\section{Materials and methods}

In the PPP both carrier-phase and pseudorange measurements are strongly recommended to be used for obtaining the highest possible point positioning accuracy. Then, we have to deal with the additional remaining unmodelled errors, namely the tropospheric delay, the satellite attitude error, and the site displacement effect. This approach is commonly known as the Precise Point Positioning, or PPP [7]. To obtain the positioning accuracy comparable to the differential positioning using only one GPS receiver, we need to use suitable software that understand the principle of the PPP technique [8].

The basic model for the dual-frequency GPS carrier-phase and pseudo-range observations from receiver $\mathrm{k}$ to satellite $\mathrm{i}$, in unit of length, is:

$$
\begin{aligned}
& L_{m_{k}^{i}}=-\lambda_{m} \phi_{m_{k}}^{i}=\varrho_{k}^{i}-\frac{\kappa}{f_{m}^{2}}+\lambda_{m} b_{m_{k}}^{i} \\
& P_{m_{k}}^{i}=\varrho_{k}^{i}+\frac{\kappa}{f_{m}^{2}}
\end{aligned}
$$

where $\phi_{m_{k}}^{i}$ and $P_{m_{k}}^{i}$ are carrier-phase and pseudo-range observations in frequency band $m$ with corresponding wave-length $\lambda_{m}$ and frequency $f_{m} ; b_{m_{k}}^{i}$ is the phase ambiguity; $\varrho_{k}^{i}$ is the nondispersive delay, including geometric delay, tropospheric delay, clock biases and any other delay which affects all the observations identically; the second term on the right side is the ionospheric delay. The multipath effect and noise are not included for clarity [9]. The receiver- and satellitedependent pseudo-range biases [10] are also ignored because the constant shifts have no substantial effect on ambiguity fixing. The carrier-phase ambiguity is composed of the following three terms:

$$
b_{m_{k}}^{i}=n_{m_{k}}^{i}+\Delta \phi_{m}^{i}-\Delta \phi_{m_{k}}
$$

where $n_{m_{k}}^{i}$ is the integer ambiguity, $\Delta \phi_{m}^{i}$ and $\Delta \phi_{m_{k}}$ are uncalibrated phase delays in the receiver and in the satellite transmitter, respectively. The uncalibrated phase delays are not integer values thus preventing the resolution of the integer ambiguities.

The tropospheric delay is commonly broken into two components, dry and wet. The dry component represents about $90 \%$ of the delay and can be predicted to a high degree of accuracy using a mathematical model, e.g., the Hopfield model. The wet component of the tropospheric delay depends on the water vapor along the GPS signal path. Unlike the dry component, the wet component is not easy to predict, and is commonly treated as an additional unknown parameter in the estimation process [11]

Precise Point Positioning (PPP) [12] [7] becomes a very pragmatic tool that reduces the computation burden for applications where co-variances among parameters of different stations are not of interest.

Normally, ionosphere-free combination observables are used in PPP to eliminate the first-order ionospheric delays in the pseudorange and carrier-phase measurements [13]. Hence, the linearized undifferenced measurement equations between receiver i and satellite $\mathrm{k}$ at a particular epoch are: 


$$
\left\{\begin{array}{c}
\Delta P_{i}^{k}=u_{i}^{k} \Delta x^{k}{ }_{i}+c \Delta t_{i}^{k}+\lambda\left(b_{i}^{k}+\delta b_{i}^{k}\right)-e_{i}^{k} \\
\Delta L_{i}^{k}=u_{i}^{k} \Delta x^{k}{ }_{i}+c \Delta t_{i}^{k}+\lambda\left(B_{i}^{k}+\delta B_{i}^{k}\right)+\lambda \Delta N_{i}^{k}-\varepsilon_{i}^{k}
\end{array}\right.
$$

where $\Delta P_{i}^{k}$ and $\Delta L_{i}^{k}$ denote the observed minus computed measurements for the pseudorange and carrier-phase, respectively; $u_{i}^{k}$ contains the unit vector from the satellite to the receiver and the mapping function of the zenith tropospheric delay (ZTD); $\Delta x^{k}{ }_{i}$ contains the increments for the a priori receiver position vector and ZTD; $c$ denotes the light speed; $\lambda$ denotes the narrow-lane wavelength, and thus following "FCBs - fractional-cycle biases" represent "narrow-lane FCBs" for brevity except when otherwise noted; $\Delta t_{i}^{k}=\Delta t_{i}-\Delta t^{k}$ where $\Delta t_{i}$ and $\Delta t^{k}$ denote the increments for the a priori receiver and satellite clocks, respectively; $B_{i}^{k}+\delta B_{i}^{k}$ is the carrierphase FCB where $B_{i}^{k}$ denotes the constant offset, i.e. the time-invariant part, and $\delta B_{i}^{k}$ denotes the time-dependent deviation from $B_{i}^{k} ; B_{i}^{k}=B_{i}-B^{k}$ and $\delta B_{i}^{k}=\delta B_{i}-\delta B^{k}$ where $B_{i}$ and $\delta B_{i}$ are for the receiver whereas $B^{k}$ and $\delta B^{k}$ are for the satellite; similarly $b_{i}^{k}+\delta b_{i}^{k}$ is the fractional-cycle part of the pseudorange bias which is hereafter called pseudorange FCB for convenience; note that integer-cycle biases do not affect the integer properties of ambiguities, and are ignored throughout this study; $\Delta N_{i}^{k}$ denotes the integer increments of the a priori narrowlane ambiguity; finally $e_{i}^{k}$ and $\varepsilon_{i}^{k}$ denote the residual errors of the pseudorange and carrier-phase measurements, respectively [14].

\section{Processing and Results}

The case study was carried out in Oradea. The data was obtained from the permanent station placed on the Faculty of Construction and Architecture. The model from the antenna calibration was LEICA GRX1200+GNSS.

Tropospheric delays were corrected using the VMF1 model with the global mapping function of [15], and the ionosphere free linear combination was used. The station coordinates and receiver clock errors were estimated epoch by epoch. Also no ocean tidal loading was used.

One of the main concerns related to PPP is the convergence time required to produce meaningful estimates. Even though the final accuracies that can be achieved with this technique are certainly very good, as shown here, the time required to achieve them (usually around several tens of minutes) is currently a bit of an impediment in the use of PPP for real-time applications [3].
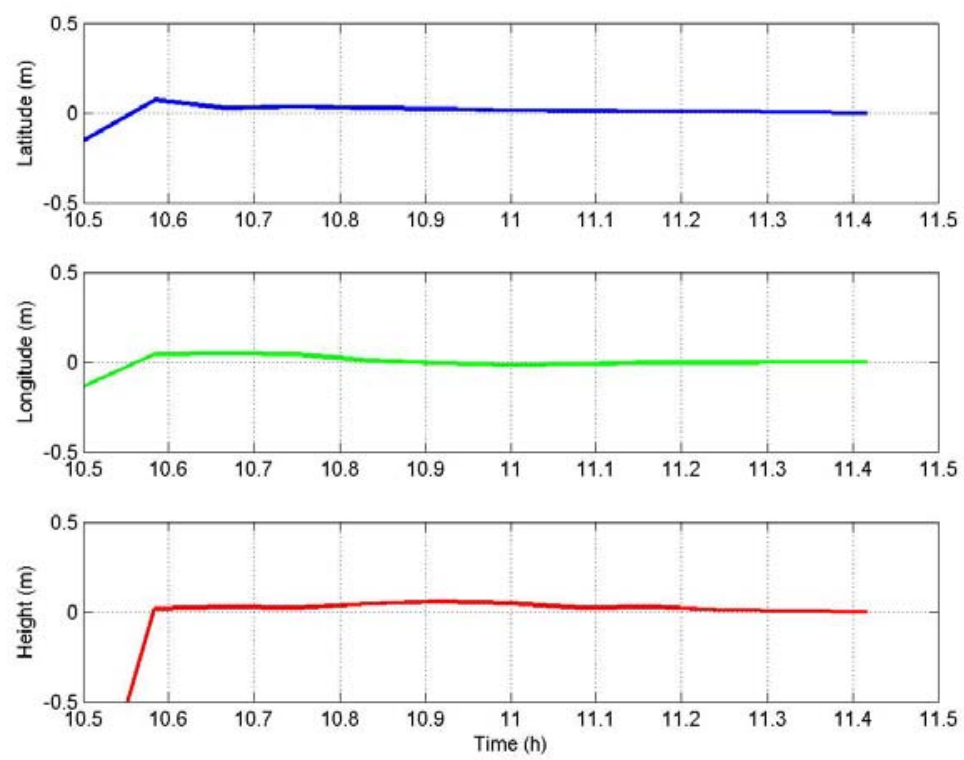

Fig. 1 - Coordinates convergence latitude, longitude and height 
The position error convergence derived from all solutions in latitude, longitude and height are presented in Fig1.

In Fig.2 it is presented the position error convergence in X,Y,Z.
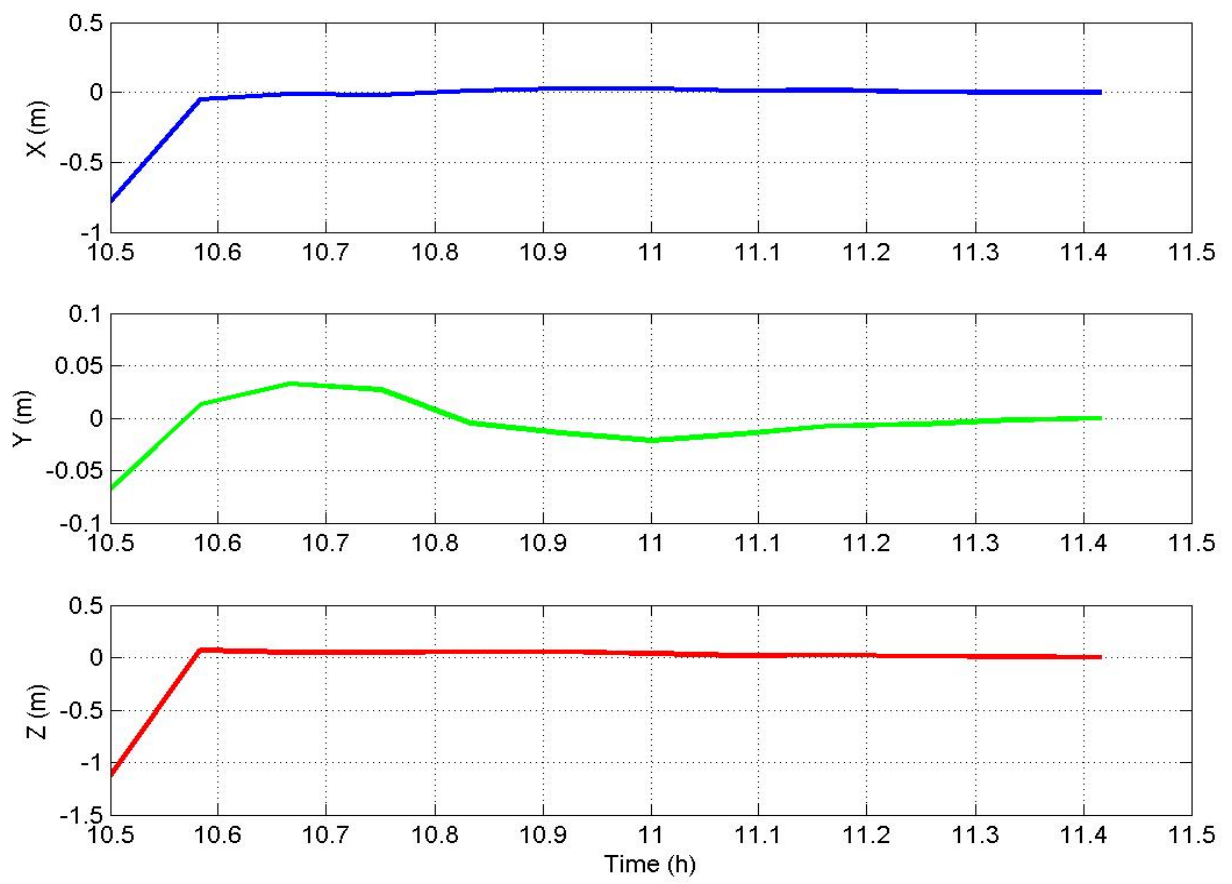

Fig. 2 - Coordinates convergence X,Y,Z

The standard deviation is presented in Fig.3.
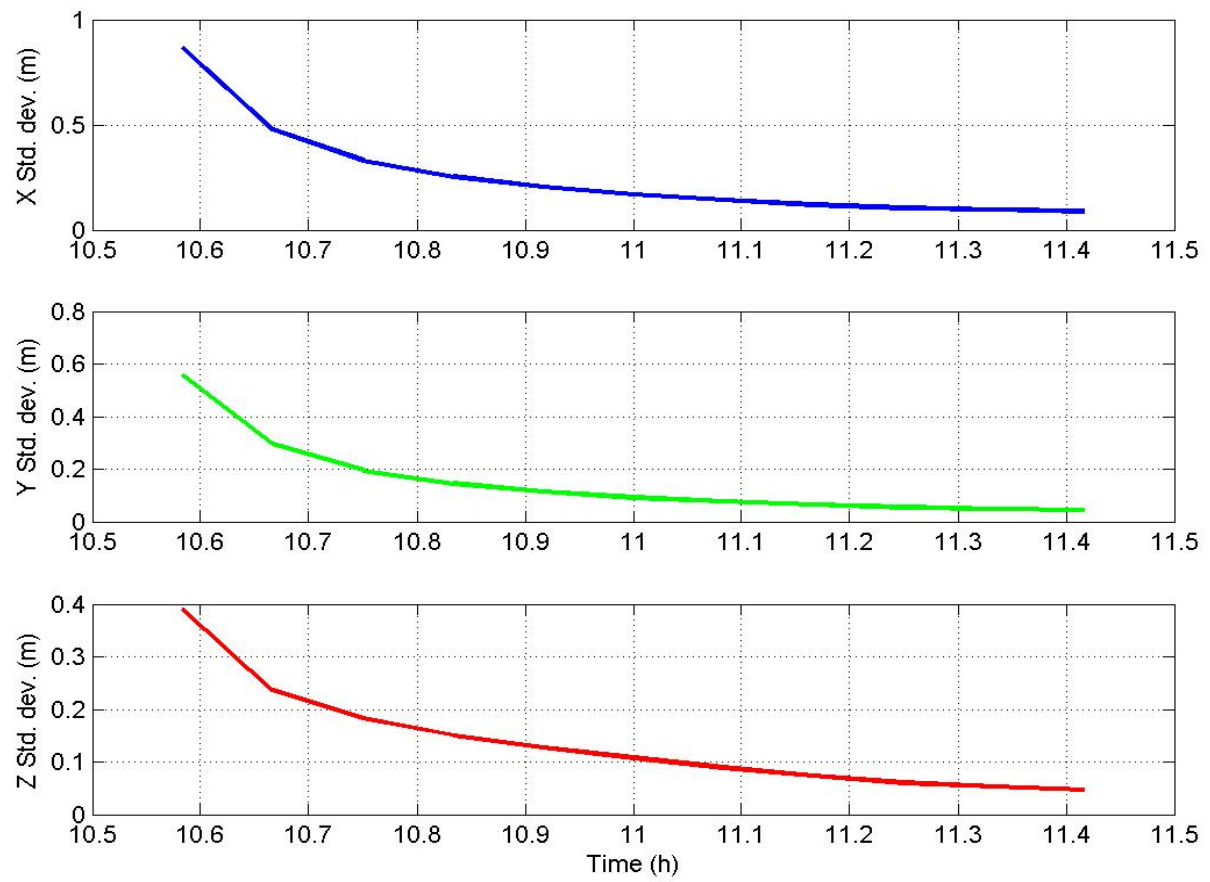

Fig. 3 - Standard deviation

The neutral atmosphere zenith delay is presented in Fig. 4 with the delay parameter that was modeled as a random walk process with a noise of $5.0 \mathrm{~mm} / \mathrm{sqrt}(\mathrm{h})$. The delay used by the model 
as an a priori value with a given uncertainty of $0.100 \mathrm{~m}$ is also shown. The elevation cutoff angle was set to $10^{0}$ which might not be low enough for a proper de-correlation.
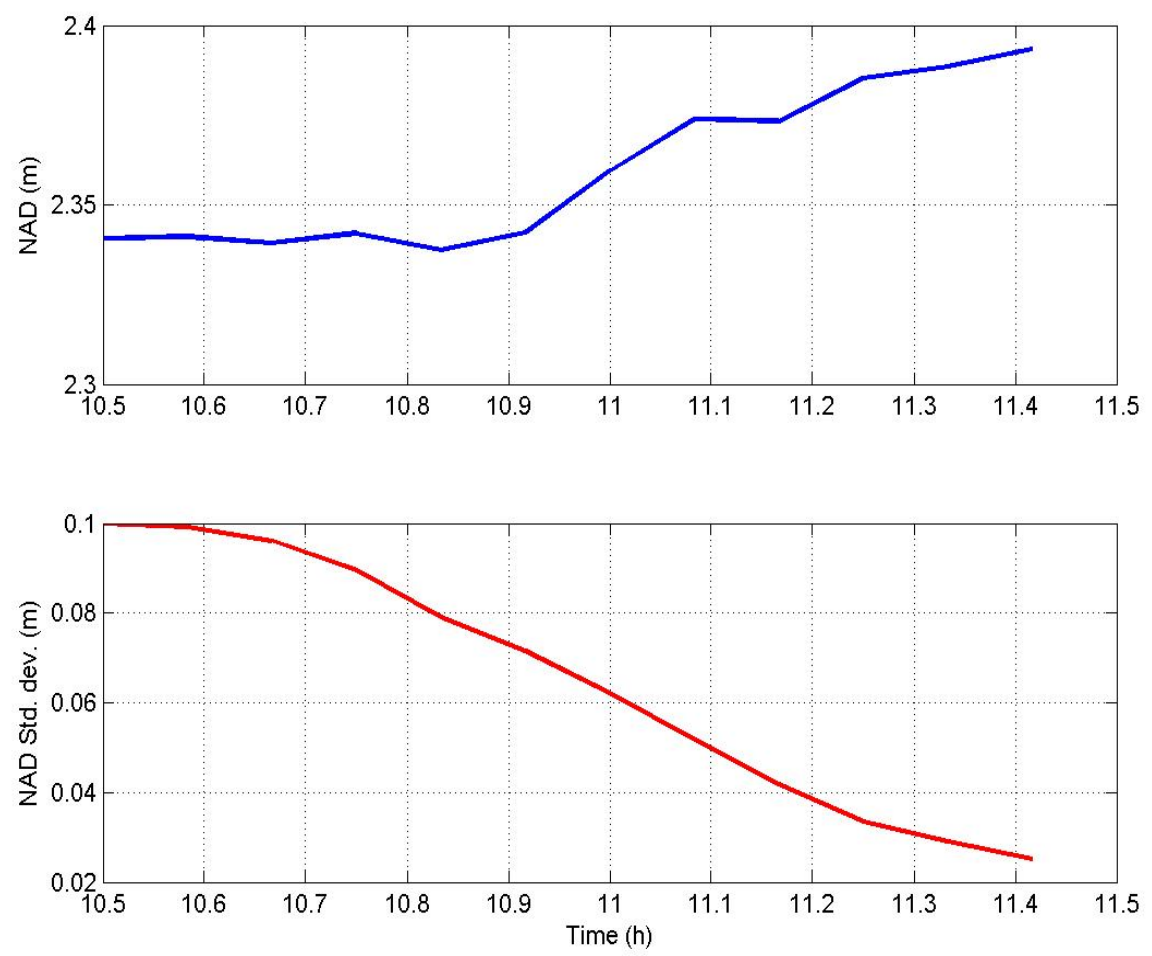

Fig. 4 - Neutral atmosphere zenith delay

The GPS receiver networks have been used for monitoring the ionosphere for some time but we have to pay attention because we use only one receiver. The filter used to estimate ionospheric delays is connected to the PPP filter that uses carrier-phase measurements to avoid unwanted effects present in code measurements. The ionospheric estimation is performed by means of a least-squares adjustment. The parameters are the ionospheric model elements (vertical delay and gradients) and the ambiguities, where the ionospheric model parameters are treated as stochastic parameters, while the ambiguities are assumed constant - thus the noise is added to them. The vertical ionospheric delay is presented in Fig.5.

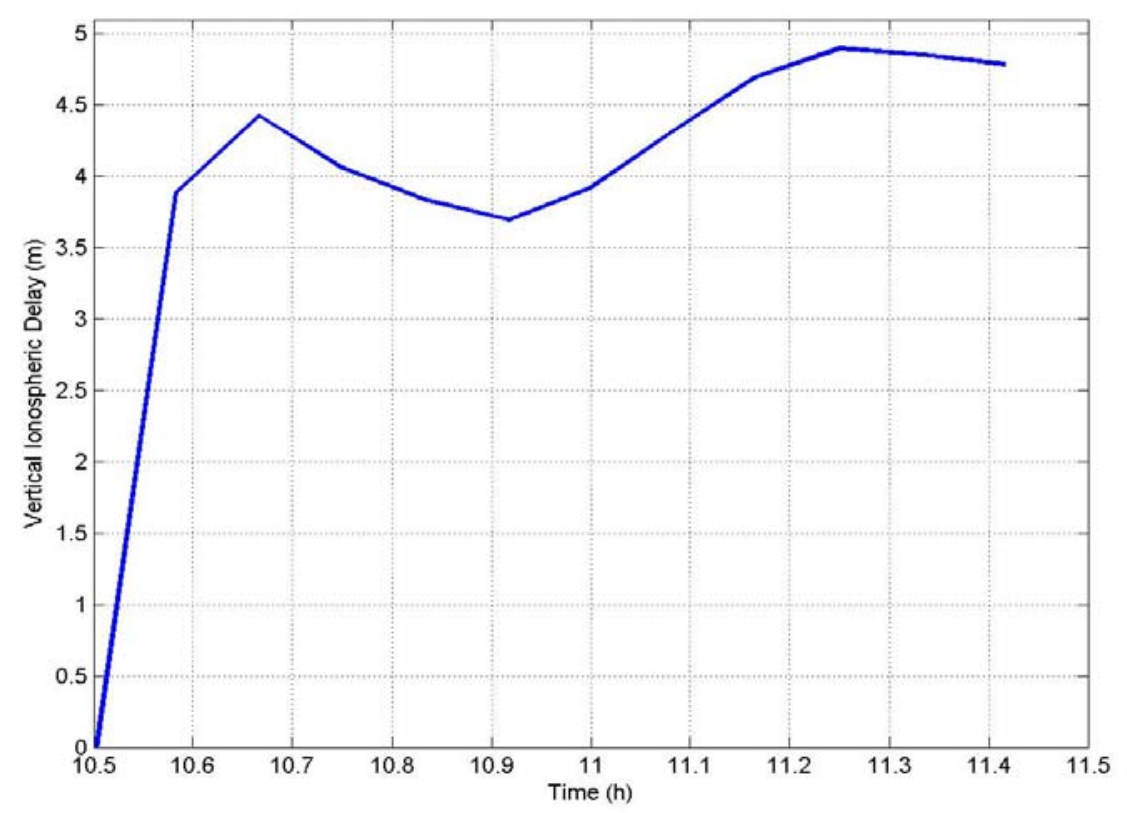

Fig. 5 - Vertical ionospheric delay 
The ionospheric model parameters are treated as stochastic parameters, while the ambiguities are assumed constant (thus no noise is added to them).

The correction for higher-order ionospheric delay when estimating the IGS precise orbit and clock are not taken into account. From this it result that the residual error component is expected to be temporally correlated.

The carrier-phase and pseudorange residual are presented in Fig.6. The residuals from the carrier-phase had values usually within $\pm 0.015 \mathrm{~m}$ resulting a reasonably stable spread of the residuals. Also the residuals from the pseudorange had values usually within $\pm 1 \mathrm{~m}$ which is also a reasonably stable spread of the residuals.
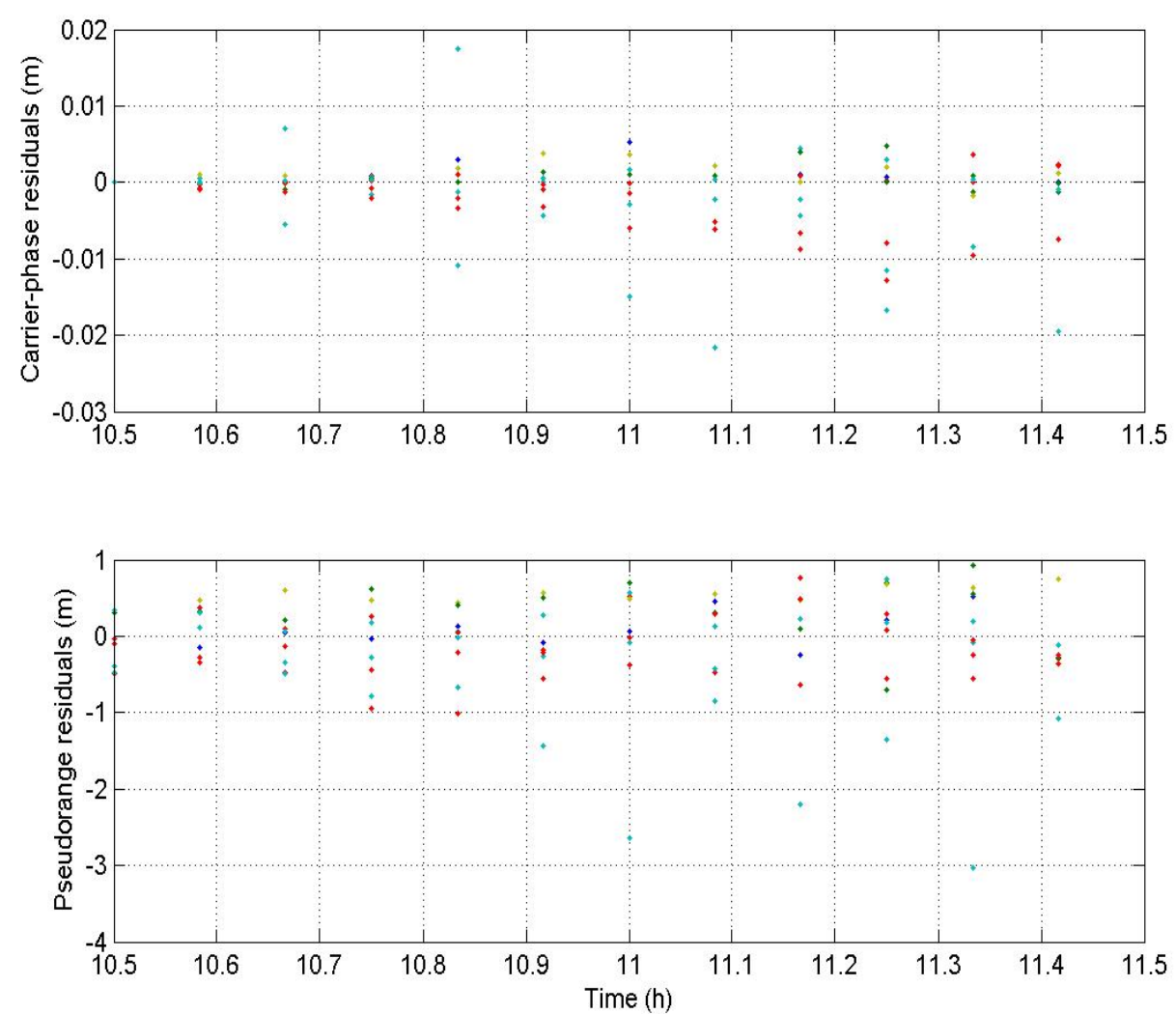

Fig. 6 - Carrier-phase and pseudorange residual

In table 2 the results are presented, from the estimated and a-priori coordinates the difference and their sigma's. The coordinates are expressed in ETRS 1989. To obtain a sufficient accuracy using the PPP technique, it is necessary to use not only the pseudorange but also the carrierphase measurements.

Table 2.

Final offsets w.r.t. a-priori coordinates

\begin{tabular}{|c|c|r|r|c|}
\hline CARTESIAN & ESTIMATED & A-PRIORI & Delta(m) & RMS(m) \\
\hline $\mathrm{X}(\mathrm{m})$ & 4037694.4169 & 4037694.9203 & -0.5034 & 0.0908 \\
\hline $\mathrm{Y}(\mathrm{m})$ & 1626553.3870 & 1626552.9865 & 0.4005 & 0.0898 \\
\hline $\mathrm{Z}(\mathrm{m})$ & 4646396.4938 & 4646396.1746 & 0.3192 & 0.0433 \\
\hline
\end{tabular}

\section{Conclusions}

GNSS positioning has become a key technology in earth sciences and civil engineering but to obtain the coordinates using the GPS technology we can use two different methods: precise the 
first method is point positioning - when we employ only one GPS receiver, and the second method is using differential (relative) positioning - when we use two or more GPS receivers simultaneously tracking the same satellites.

Recent progress achieved in the PPP ambiguity resolution can improve the positioning accuracy to approximately $0.5 \mathrm{~cm}$ in the horizontal plane and about $1.5 \mathrm{~cm}$ in the vertical direction even when only hourly observations are used. Nevertheless, correct ambiguity resolution does not always lead to an improved accuracy of a position estimate.

Therefore, the PPP-based approach does offer an alternative way to determine the geodetic coordinates at a slightly lower accuracy than the 'conventional' way, but due to electronic advancement and the development of robust algorithms we can expect a significant improvement.

\section{References}

[1]. King M., Edwards S., and Clarke P. (2002). Precise point positioning: Breaking the monopoly of relative GPS processing. Eng. Surv. Showc., pp. 40-41.

[2]. Wabbena G., Schmitz M., and Bagge A.(2005). PPP-RTK: Precise Point Positioning Using State-Space Representation in RTK Networks, in Proceedings of the 18th International Technical Meeting of the Satellite Division of The Institute of Navigation (ION GNSS 2005), pp. 2584-2594.

[3]. Leandro R.F., Santos M. C., and Langley R. B. (2010). Analyzing GNSS data in precise point positioning software, GPS Solut., vol. 15, no. 1, pp. 1-13.

[4]. Rizos C. (2008). The Research Challenges of IAG Commission 4 'Positioning \& Applications, in VI HotineMarussi Symposium on Theoretical and Computational Geodesy SE - 19, vol. 132, P. Xu, J. Liu, and A. Dermanis, Eds. Springer Berlin Heidelberg, pp. 126-132.

[5]. Blewitt G. (1989). Carrier phase ambiguity resolution for the Global Positioning System applied to geodetic baselines up to 2000 km, J. Geophys. Res. Solid Earth, vol. 94, no. B8, pp. 10187-10203.

[6]. Xu P., Shi C., Fang R., Liu J., Niu X., Zhang Q. and Yanagidani T.(2012). High-rate precise point positioning (PPP) to measure seismic wave motions: an experimental comparison of GPS PPP with inertial measurement units,J. Geod., vol. 87, no. 4, pp. 361-372.

[7]. Héroux P. and Kouba J. (2001). GPS precise point positioning using IGS orbit products, Phys. Chem. Earth, Part A Solid Earth Geod., vol. 26, no. 6, pp. 573-578.

[8]. Nistor S. and Buda A. S. (2015). Ambiguity resolution in precise point positioning technique: a case study, J. Appl. Eng. Sci.

[9]. Ge M., Gendt G., Rothacher M., Shi C., and Liu J.(2007). Resolution of GPS carrier-phase ambiguities in Precise Point Positioning (PPP) with daily observations, J. Geod., vol. 82, no. 7, pp. 389-399.

[10]. Schaer S. and Steigenberger P. (2006). Determination and use of GPS differential code bias values, in IGS Workshop, pp. 8-11.

[11]. El-Rabbany A.(2002), Precise GPS point positioning: the future alternative to differential GPS surveying, Dept. Civ. Eng. (Geomatics Option), Ryerson Univ.

[12]. Zumberge J.P., Heflin M. B., Jefferson D. C., Watkins M. M., and Webb F. H. (1997). Precise point positioning for the efficient and robust analysis of GPS data from large networks, J. Geophys. Res., vol. 102, no. B3, p. 5005.

[13]. Geng J., Meng X., Dodson A. H., and Teferle F. N. (2010). Integer ambiguity resolution in precise point positioning: method comparison, J. Geod., vol. 84, no. 9, pp. 569-581.

[14]. Teunissen P. G. (1998). GPS Carrier Phase Ambiguity Fixing Concepts, in GPS for Geodesy SE - 8, Teunissen P. G. and Kleusberg A., Eds. Springer Berlin Heidelberg, pp. 319-388.

[15]. Boehm J., Werl B., and Schuh H.(2006). Troposphere mapping functions for GPS and very long baseline interferometry from European Centre for Medium-Range Weather Forecasts operational analysis data, $J$. Geophys. Res., vol. 111, no. B2, p. B02406. 